\title{
Publisher Correction: Approaches for periodic inventory control under random production yield and fixed setup cost
}

\author{
G. P. Kiesmüller ${ }^{1}$ • K. Inderfurth ${ }^{1}$
}

Published online: 24 January 2018

(C) Springer-Verlag GmbH Germany, part of Springer Nature 2018

\section{Correction to: OR Spectrum https://doi.org/10.1007/s00291-017-0489-8}

In the original version of the article, operator symbols " $\rfloor$ " " $\rceil$ " have been inadvertently processed as square brackets in Eqs. 17, 20, the first line of the third paragraph and second line of the fourteenth paragraph in Sect. 4.1.

$$
I_{t}=S-D(\lambda+t)+\sum_{k=1}^{\lceil\lambda / R\rceil}\left\{Y\left(Q_{k}\right)-E\left[Y\left(Q_{k}\right)\right]\right\} \quad t=1,2, \ldots, R
$$

Here, $\lceil\lambda / R\rceil$ describes the number of open orders in a replenishment cycle of $R$ periods.

$$
\sigma_{I, t}^{2}(R, S)=(\lambda+t) \sigma_{D}^{2}+\lceil\lambda / R\rceil \frac{\rho_{Z}^{2}\left(R \sigma_{D}^{2}+R^{2} \mu_{D}^{2}\right)}{\left(1-\rho_{Z}^{2}\right)} t=1,2, \ldots, R .
$$

Since up to $(\lambda-1)$ replenishments with unknown yield deviations can occur during the risk period, on average risks from $\lfloor(\lambda-1) / R\rfloor$ deviations have to be taken into account if the duration of a replenishment cycle is $R$ periods.

The original article has been corrected.

The original article can be found online at https://doi.org/10.1007/s00291-017-0489-8.

G. P. Kiesmüller

gudrun.kiesmueller@ovgu.de

K. Inderfurth

karl.inderfurth@ovgu.de

1 Otto von Guericke University Magdeburg, Universitätsplatz 2, 39106 Magdeburg, Germany 